\title{
Prediction of two-dimensional diluted magnetic semiconductors: Doped monolayer $\mathrm{MoS}_{2}$ systems
}

\author{
Y. C. Cheng, ${ }^{1}$ Z. Y. Zhu, ${ }^{1}$ W. B. Mi, ${ }^{2}$ Z. B. Guo, ${ }^{3}$ and U. Schwingenschlögl ${ }^{1, *}$ \\ ${ }^{1}$ PSE Division, King Abdullah University of Science and Technology (KAUST), Thuwal 23955-6900, Kingdom of Saudi Arabia \\ ${ }^{2}$ Tianjin Key Laboratory of Low Dimensional Materials Physics and Preparing Technology, Institute of Advanced Materials Physics, \\ Faculty of Science, Tianjin University, Tianjin 300072, China \\ ${ }^{3}$ Core Labs, King Abdullah University of Science and Technology (KAUST), Thuwal 23955-6900, Kingdom of Saudi Arabia
}

(Received 12 September 2012; published 5 March 2013)

\begin{abstract}
Using first-principles calculations, we propose a two-dimensional diluted magnetic semiconductor: monolayer $\mathrm{MoS}_{2}$ doped by transition metals. Doping of transition metal atoms from the IIIB to VIB groups results in nonmagnetic states, since the number of valence electrons is smaller or equal to that of Mo. Doping of atoms from the VIIB to IIB groups becomes energetically less and less favorable. Magnetism is observed for Mn, $\mathrm{Fe}, \mathrm{Co}, \mathrm{Zn}, \mathrm{Cd}$, and $\mathrm{Hg}$ doping, while for the other dopants from these groups it is suppressed by Jahn-Teller distortions. Analysis of the binding energies and magnetic properties indicates that $(\mathrm{Mo}, X) \mathrm{S}_{2}(X=\mathrm{Mn}, \mathrm{Fe}, \mathrm{Co}$, and $\mathrm{Zn}$ ) are promising systems to explore two-dimensional diluted magnetic semiconductors.
\end{abstract}

DOI: 10.1103/PhysRevB.87.100401

PACS number(s): 75.50.Pp, 73.22.-f, 75.75.-c

In the past years extensive investigations of diluted magnetic semiconductors (DMSs) have been performed, because of exciting effects in this materials class, such as exciton spin polarization and spin-polarized electrical current, which gives rise to potential applications in spintronics. ${ }^{1,2}$ Research has focused on the transition metal (transition metal) doped III-V and II-VI three-dimensional (3D) semiconductors. On the other hand, two-dimensional (2D) materials have raised a lot of attention recently, in particular, since the discovery of graphene in 2004. ${ }^{3}$ This also brought up the idea of 2D DMSs. ${ }^{4,5}$ Though graphene has been considered as a promising host material for transition metal implantation, ${ }^{6}$ the lack of a band gap hampers the control of the charge and magnetic states. It has been proposed that this difficulty can be overcome by the introduction of semiconducting layers, such as monolayer $h$-BN. ${ }^{7}$ However, as a consequence of the single atom thickness of both graphene and $h-\mathrm{BN}$ as well as optimization of the bond length to the implanted transition metal atoms, ${ }^{6,7}$ the latter will stick out of the plane of the host material, which leads to chemical instability if the system is exposed to air.

The semiconducting transition metal dichalcogenides $M X_{2}$ ( $M=\mathrm{Mo}, \mathrm{W}$ and $X=\mathrm{S}, \mathrm{Se})$ crystallize in a layered $2 H$ prototype structure consisting of weakly van der Waals bonded $X-M-X$ slabs. They are characterized by distinct electronic, optical, and catalytic properties. ${ }^{8-10}$ The compounds are also useful for dry lubrication ${ }^{11}$ and exhibit interesting valley physics. ${ }^{12}$ Recently, monolayer $\mathrm{MoS}_{2}$ (1.8 eV direct band gap) has been fabricated by micromechanical exfoliation, ${ }^{13}$ liquid exfoliation, ${ }^{14}$ and chemical vapor deposition. ${ }^{15}$ In addition, monolayer $\mathrm{MoS}_{2}$ transistors have been demonstrated with carrier mobilities of $200 \mathrm{~cm}^{2} \mathrm{~V}^{-1} \mathrm{~s}^{-1}$ at room temperature (which is similar to that of graphene nanoribbons), current on/off ratios of $1 \times 10^{8}$, and ultralow standby power dissipation. ${ }^{14}$ Because of the sandwich S-Mo-S structure [see Fig. 1(a)], the direct band gap of $1.8 \mathrm{eV}$, and the transition metal nature of Mo, we expect to obtain a 2D DMS by replacing Mo by other transition metal atoms to induce electron or hole doping and magnetism.

In this Rapid Communication, we present a systematic first-principles study of monolayer $\mathrm{MoS}_{2}$ doped by transition metal atoms from periods four, five, and six. The relaxed structures for dopants from the IIIB to VIB groups retain $C_{3 v}$ symmetry, while dopants from the VIIB to IIB groups (except for $\mathrm{Mn}, \mathrm{Fe}, \mathrm{Co}, \mathrm{Zn}, \mathrm{Cd}$, and $\mathrm{Hg}$ ) lead to a Jahn-Teller distortion. As a consequence, there is no magnetism in these systems. We propose that 2D DMSs are very likely to be realized in monolayer $\mathrm{MoS}_{2}$ doped by $\mathrm{Mn}, \mathrm{Fe}, \mathrm{Co}$, or $\mathrm{Zn}$.

We address the electronic properties of doped monolayer $\mathrm{MoS}_{2}$ using the QUANTUM ESPRESSO package in the framework of density functional theory. ${ }^{16,17}$ Ultrasoft pseudopotentials ${ }^{18}$ as well as the generalized gradient approximation in the Perdew-Burke-Ernzerhof parametrization ${ }^{19}$ of the exchange correlation functional are employed. All simulations are carried out for a $4 \times 4 \times 1$ supercell with 16 Mo atoms and $32 \mathrm{~S}$ atoms. A $10 \AA$ vacuum layer is adopted in our slab calculations to avoid interaction between the slabs. ${ }^{20}$ By replacing one Mo atom with a transition metal atom, the impurity concentration is set to $6.25 \%$. A high cutoff energy of $544 \mathrm{eV}$ and a precise $4 \times 4 \times 1 k$-point sampling grid are used. For the self-consistency cycle we employ an energy criterion of $10^{-10} \mathrm{eV}$. The structural optimization is continued until the residual forces have converged to less than $2.6 \times 10^{-3} \mathrm{eV} / \AA$ and the total energy to less than $1.4 \times 10^{-4} \mathrm{eV}$. We also have adopted on-site Coulomb interactions ranging from 1 to $4 \mathrm{eV}$ to study the effects on the structure and magnetic properties of $\mathrm{Ni}$ and $\mathrm{Mn}$ doped monolayer $\mathrm{MoS}_{2}$. However, we find no relevant influence on our conclusions and therefore discuss in the following the results without on-site interaction.

Bulk $\mathrm{MoS}_{2}$ has a layered $2 \mathrm{H}$ prototype structure with space group $\mathrm{P}_{3} m m c$ ( $D_{6 h}$ point group), which consists of weakly van der Waals bonded S-Mo-S slabs, and an indirect band gap of $1.3 \mathrm{eV}$. The trigonal prismatic coordination of the bulk is maintained in monolayer $\mathrm{MoS}_{2}$, whereas the symmetry is reduced to $P \overline{6} m 2$ ( $D_{3 h}$ point group) due to a loss of inversion symmetry. Figure 1(a) shows a side view of the atomic structure of monolayer $\mathrm{MoS}_{2}$, which is a semiconductor with a direct band gap of $1.8 \mathrm{eV} .^{10,21,22}$ The valence band maximum and conduction band minimum are located at the $K$ point of the Brillouin zone. The point group of monolayer $\mathrm{MoS}_{2}$ with one Mo vacancy remains $C_{3 v}$ [see Fig. 1(b)]. The same applies to the replacement of one Mo atom by another transition metal atom [see Fig. 1(c)]. Under the structure relaxation the $C_{3 v}$ 
(a)

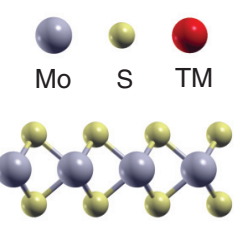

(c)

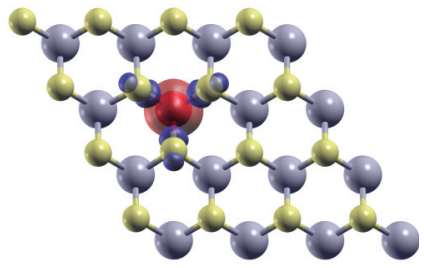

FIG. 1. (Color online) Monolayer $\mathrm{MoS}_{2}$ : (a) Side view, (b) top view with Mo vacancy, (c) spin density for Mn doping with $C_{3 v}$ symmetry, and (d) Ni doping with $C_{1}$ symmetry. Spin densities of $\pm 0.002 \mathrm{e} / \mathrm{bohr}^{-3}$ are visualized by red and blue isosurfaces, respectively.

symmetry is maintained for dopants from the IIIB to VIB and IIB groups as well as $\mathrm{Mn}, \mathrm{Fe}$, and Co, whereas a Jahn-Teller distortion is observed otherwise.

Though the formation of a Mo vacancy requires more energy than that of a $S$ vacancy, it has been reported that Co doping can be achieved at the $\mathrm{MoS}_{2}$ edge by sulfidation of a mixture of ammonium heptamolybdate and cobalt nitrate, ${ }^{23}$ which paves the way to transition metal doping by substitution of Mo in $\mathrm{MoS}_{2}$. We have studied a zigzag $\mathrm{MoS}_{2}$ nanoribbon with one $\mathrm{S}$ edge and one Mo edge to mimic the reported edge structure. Due to the dangling bonds at the edges, the nanoribbon turns out to be ferromagnetic with a total magnetic moment of $2.5 \mu_{B}$ per cell. The moment reduces to $1.3 \mu_{B}$ per cell under substitution of one Mo on the Mo edge by Co, which can be attributed to the coupling of the Co moment to the edge state.

Moreover, it has been demonstrated experimentally that pulsed laser deposition can be used to dope graphene by single transition metal atoms, such as $\mathrm{Pt}, \mathrm{Co}$, and $\mathrm{In} .{ }^{24}$ It is to be expected that the same approach can be applied to monolayer $\mathrm{MoS}_{2}$ to realize single atom doping. To check the stability of different dopants, we have calculated the binding energies as summarized in Fig. 2. The binding energy is defined as $E_{b}=E_{v}+\mu-E_{d}$, where $E_{v}$ is the energy of a relaxed $\mathrm{MoS}_{2}$ monolayer with one Mo vacancy, $\mu$ is the energy of the doped transition metal atom, and $E_{d}$ is the energy of a relaxed $\mathrm{MoS}_{2}$ monolayer with one Mo atom replaced by the transition metal.
The value of $E_{b}$ for different dopants varies strongly from 0.2 to $16.0 \mathrm{eV}$. Mo self-substitution yields the binding energy of a Mo atom, which is $13.5 \mathrm{eV}$. The three values of $E_{b}$ shown in Fig. 2 for the IVB, VB, and VIB groups are similar, and larger than for the other groups. The binding energy increases with the period for the IVB, VB, VIB, VIII8, and VIII9 groups, while it decreases for the VIII10, IB, and IIB groups. We can conclude that substitutional doping of transition metal atoms from the IIIB to VIII10 groups is stable. Doping of transition metal atoms from the IB and IIB groups is difficult because of the low binding energies (especially for $\mathrm{Hg}$ ). We find that monolayer $\mathrm{MoS}_{2}$ doped by $\mathrm{Mn}, \mathrm{Fe}, \mathrm{Co}, \mathrm{Zn}, \mathrm{Cd}$, or $\mathrm{Hg}$ is ferromagnetic. The magnetic moments are listed in Table I. The binding energies and magnetic moments indicate that $\mathrm{Mn}$, $\mathrm{Fe}, \mathrm{Co}$, and $\mathrm{Zn}$ doping of monolayer $\mathrm{MoS}_{2}$ are very promising approaches to achieve 2D DMSs. The origin of the magnetism for $\mathrm{Mn}, \mathrm{Fe}, \mathrm{Co}, \mathrm{Zn}, \mathrm{Cd}$, and $\mathrm{Hg}$ doping as well as the absence of magnetism in the other cases will be discussed later.

The density of states (DOS) in Fig. 3(a) shows a band gap of $1.70 \mathrm{eV}$ for the pristine monolayer $\mathrm{MoS}_{2}$, which is only an underestimation of $0.10 \mathrm{eV}$ as compared to the values obtained experimentally ${ }^{10}$ and by $G W$ calculations. ${ }^{22}$ The tiny difference indicates the validity of the generalized gradient approximation for $\mathrm{MoS}_{2}$. For $\mathrm{S}$ vacancies in $\mathrm{MoS}_{2}$, Fig. 3(b) shows unoccupied shallow impurity states in the band gap near the conduction bands. In addition, the formation energy of a $S$ vacancy is $6.0 \mathrm{eV}$, which is much less than that of a Mo vacancy $(13.5 \mathrm{eV})$. In fact, it has been demonstrated that a $\mathrm{S}$ vacancy is easier formed under electron irradiation than a Mo vacancy. ${ }^{25}$ This also demonstrated that the vacancies can be consecutively filled with other atomic species. For example, $\mathrm{F}, \mathrm{Cl}, \mathrm{Br}$, and I can donate electrons to the $\mathrm{MoS}_{2}$ and fill the unoccupied shallow impurity states near the conduction bands. ${ }^{25}$ Both the electronic structure and formation energies indicate that the filling of a $\mathrm{S}$ vacancy with a donor atom induces free electrons in monolayer $\mathrm{MoS}_{2} .{ }^{13}$

Before turning to $\mathrm{MoS}_{2}$ doped by transition metal atoms, we next study the electronic structure of monolayer $\mathrm{MoS}_{2}$ with one Mo vacancy. The structure maintains the $C_{3 v}$ symmetry [see Fig. 1(b)]. There are states in the band gap near the valence bands [see Fig. 3(c)]. The defect levels can be classified as $a$, $e_{1}$, and $e_{2}$ states due to a mixture of the $\mathrm{S} p$ orbitals. Each Mo vacancy can extract four electrons from the system, which results in $p$-type doping. Two electrons occupy the $e_{1}$ spin-up channel and two occupy the $e_{1}$ spin-down channel. Therefore, neither Mo vacancies nor $\mathrm{S}$ vacancies can introduce magnetism in $\mathrm{MoS}_{2}$, which is different from the intrinsic magnetism in defective graphene and $h$-BN. . $, 7,26$

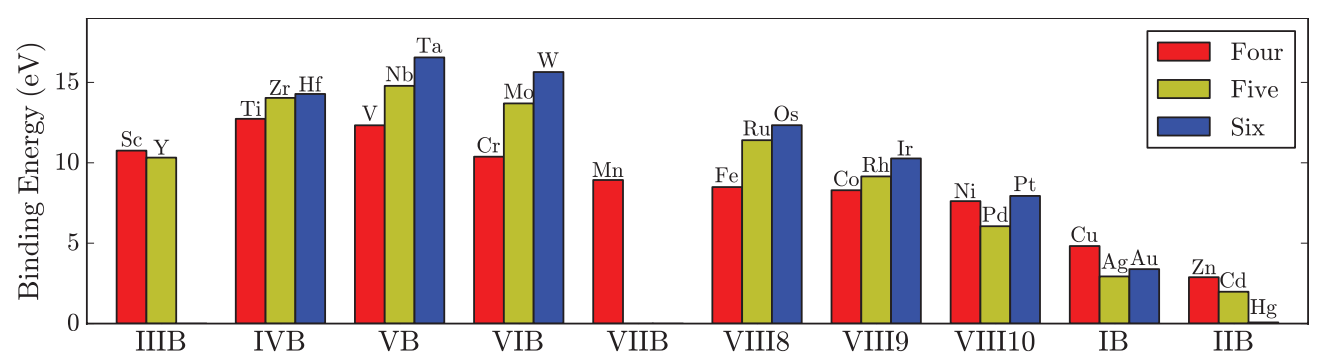

FIG. 2. (Color online) Binding energies of monolayer $\mathrm{MoS}_{2}$ doped by transition metal atoms from the periods four, five, and six. 
TABLE I. Magnetic moment, crystal field splitting $\Delta_{\mathrm{cf}}$, exchange splitting $\Delta_{\mathrm{ex}}$, magnetic energy $\Delta E=E_{\mathrm{FM}}-E_{\mathrm{AFM}}$, and Curie temperature $T_{C}$ for $(\mathrm{Mo}, X) \mathrm{S}_{2}(X=\mathrm{Mn}, \mathrm{Fe}, \mathrm{Co}, \mathrm{Zn}, \mathrm{Cd}$, and $\mathrm{Hg})$ with $6.25 \%$ doping.

\begin{tabular}{|c|c|c|c|c|c|}
\hline \multicolumn{2}{|c|}{ Magnetic moment $\left(\mu_{B}\right)$} & \multirow{2}{*}{$\frac{\Delta_{\mathrm{cf}}(\mathrm{meV})}{694}$} & \multirow{2}{*}{$\frac{\Delta_{\text {ex }}(\mathrm{meV})}{532}$} & \multirow{2}{*}{$\frac{\Delta E(\mathrm{meV})}{-0.02}$} & \multirow{2}{*}{$\frac{T_{C}(\mathrm{~K})}{0.2}$} \\
\hline $\mathrm{Mn}$ & 1.0 & & & & \\
\hline $\mathrm{Fe}$ & 2.0 & 704 & 650 & 3.92 & \\
\hline Co & 3.0 & 412 & 515 & 1.38 & \\
\hline $\mathrm{Zn}$ & 2.0 & 309 & 82 & -3.30 & 38.3 \\
\hline $\mathrm{Cd}$ & 2.0 & 367 & 91 & -2.88 & 33.4 \\
\hline $\mathrm{Hg}$ & 2.0 & 302 & 89 & -1.85 & 21.5 \\
\hline
\end{tabular}

With respect to the structure and calculated magnetism of (Mo, $X) \mathrm{S}_{2}$, we classify the transition metals into three classes: IIIB to VIB groups, VII8 to IB groups, and the IIB group. We take $\mathrm{Sc}$ as a prototypical element from the first class. The symmetry of (Mo,Sc) $S_{2}$ is $C_{3 v}$ and the DOS shows spin degeneracy [see Fig. 3(d)]. An isolated Sc atom has a $3 d 4 s^{2}$ configuration and three valence electrons less than Mo. The fact that the impurity states are close to the valance bands indicates $p$-type doping.

We study (Mo,Mn) $\mathrm{S}_{2}$ as a prototypical example from the second class. Figure 3(e) shows spin polarization for this case. An isolated $\mathrm{Mn}$ atom has a $3 d^{5} 4 s^{2}$ configuration with one additional valence electron as compared to Mo. The impurity states are close to the conduction bands, which indicates $n$-type doping. The spin density isosurface in Fig. 1(c) illustrates that the magnetic moment is mainly due to the $\mathrm{Mn}$ atom. The $\mathrm{Mn}$

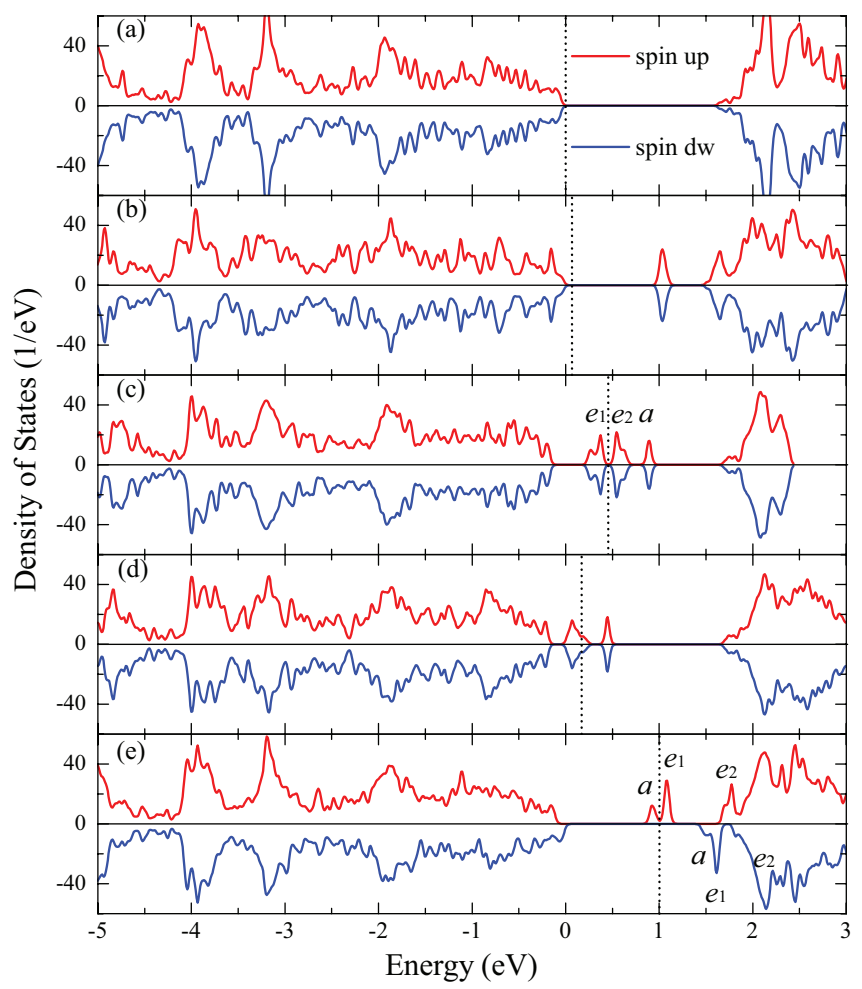

FIG. 3. (Color online) DOS of monolayer $\mathrm{MoS}_{2}$ : (a) Pristine, (b) with two S vacancies, (c) with one Mo vacancy, (d) Sc doped, and (e) Mn doped. The energy axis is calibrated with respect to the core states. The dashed vertical lines indicate the Fermi levels.
$3 d$ orbitals are split into a single $a\left(d_{z^{2}}\right)$ state and two twofold degenerate $e_{1}\left(d_{x y, x^{2}-y^{2}}\right)$ and $e_{2}\left(d_{x z, y z}\right)$ states, because of the $C_{3 v}$ symmetry. Six electrons occupy the valence states and the remaining the $a$ spin-up defect state. The exchange splitting of the $e$ orbitals, $\Delta_{\mathrm{ex}}=532 \mathrm{meV}$, is smaller than the crystal field splitting, $\Delta_{\text {cf }}=694 \mathrm{meV}$. For Fe (Co) doped $\mathrm{MoS}_{2}$, one electron occupies the $a$ defect state and the other one (two) occupy the $e_{1}$ spin-up defect state. We obtain $\Delta_{\mathrm{ex}}=650 \mathrm{meV}$ and $\Delta_{\mathrm{cf}}=704 \mathrm{meV}$ for $(\mathrm{Mo}, \mathrm{Fe}) \mathrm{S}_{2}$ and $\Delta_{\mathrm{ex}}=515 \mathrm{meV}$ and $\Delta_{\mathrm{cf}}=412 \mathrm{meV}$ for $(\mathrm{Mo}, \mathrm{Co}) \mathrm{S}_{2}$.

Figure $4(\mathrm{a})$ shows the DOS of $(\mathrm{Mo}, \mathrm{Ni}) \mathrm{S}_{2}$ with $C_{3 v}$ symmetry. The $a$ state shifts above the $e_{2}$ state so that two electrons occupy the $e_{1}$ state and two the $e_{2}$ spin-up state. However, structure relaxation shows that the symmetry of (Mo,Ni) $\mathrm{S}_{2}$ is reduced to $C_{1}$ due to a Jahn-Teller distortion [see Fig. 1(d)]. The energy difference of the $C_{3 v}$ and $C_{1}$ structures is $383 \mathrm{meV}$. Due to the Jahn-Teller distortion, we have five $a$ states. Therefore, four electrons occupy the $a_{1}$ and $a_{2}$ states [see Fig. 4(b)]. All transition metals from the second class, except for Mn, Fe, and Co, show a Jahn-Teller distortion and consequently no magnetism.

Though the binding energy for elements from the third class is lower than for the other two classes (see Fig. 2), $(\mathrm{Mo}, X) \mathrm{S}_{2}$ $(X=\mathrm{Zn}, \mathrm{Cd}, \mathrm{Hg}$ ) develops magnetism with magnetic moments of $2.0 \mu_{B}$. Figure 4(c) shows the DOS for (Mo,Zn) $S_{2}$ in $C_{3 v}$ symmetry, which is the ground state. The $e_{1}$ state is located at the top of the valence band and the $a$ state shifts above the $e_{2}$ state. As a consequence, the degenerate $e_{1}$ states

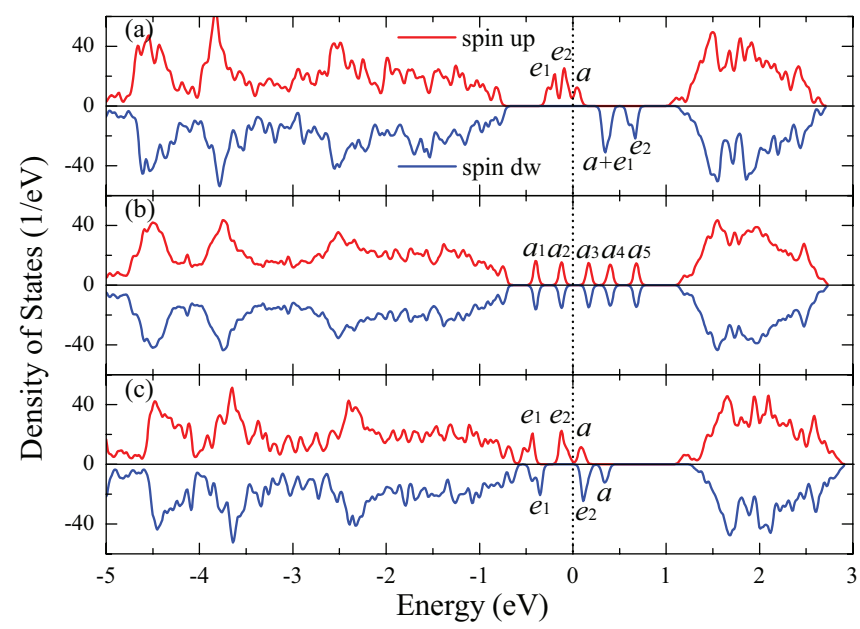

FIG. 4. (Color online) DOS of (Mo,Ni) $\mathrm{S}_{2}$ in (a) $C_{3 v}$ and (b) $C_{1}$ symmetry. (c) DOS of $(\mathrm{Mo}, \mathrm{Zn}) \mathrm{S}_{2}$ in $C_{3 v}$ symmetry. 
are occupied by four electrons and two more electrons occupy the $e_{2}$ spin-up states. As the impurity states are near the valence bands we obtain $p$-type doping.

To find the magnetic ground state, both ferromagnetic and antiferromagnetic coupling between the dopants (concentration $6.25 \%$ ) are considered for the transition metals $\mathrm{Mn}, \mathrm{Fe}, \mathrm{Co}, \mathrm{Zn}, \mathrm{Cd}$, and $\mathrm{Hg}$. For $\mathrm{Fe}$ and $\mathrm{Co}$ doping the antiferromagnetic state is energetically favorable, while $\mathrm{Mn}$, $\mathrm{Zn}, \mathrm{Cd}$, and $\mathrm{Hg}$ doping results in ferromagnetic ground states, which are $0.02,3.30,2.88$, and $1.85 \mathrm{meV}$ lower in energy than the respective antiferromagnetic states. An important parameter of a DMS is the Curie temperature $\left(T_{C}\right)$, below which the system develops long range ferromagnetic ordering. We estimate $T_{C}$ based on the mean-field theory and Heisenberg model using the relation $k_{B} T_{C}=(2 / 3) \Delta E .^{27,28}$ The results are given in Table I. It has been proposed that the typical $\mathrm{T}_{C}$ for quasi-2D DMS heterostructures (such as $\mathrm{Ga}_{1-x} \mathrm{Mn}_{x} \mathrm{As}$ ) is far below room temperature $\left(T_{C}<100 \mathrm{~K}\right)$ based on a disordered Ruderman-Kittel-Kasuya-Yosida (RKKY) lattice field theory. ${ }^{4,5}$ Our current results of $T_{C}$ are consistent with this proposal. For Mn we have repeated the calculations for a smaller dopant concentration of $4 \%$ and find a significantly enhanced Curie temperature of $T_{C}=92.7 \mathrm{~K}$.

In conclusion, we have employed first-principles calculations to study the structural, electronic, and magnetic properties of monolayer $\mathrm{MoS}_{2}$ for doping with various transition metals. Our results demonstrate that 2D DMS systems can be realized by this approach. Particularly promising is doping of monolayer $\mathrm{MoS}_{2}$ by $\mathrm{Mn}, \mathrm{Fe}, \mathrm{Co}$, and $\mathrm{Zn}$. The conclusions obtained for $\mathrm{MoS}_{2}$ should be valid also for other semiconducting transition metal dichalcogenides. Investigation of further materials from this large family therefore is promising to find new 2D DMSs with a high Curie temperature.

The computational resources for this study have been provided by KAUST IT.
${ }^{*}$ Udo.Schwingenschlogl@ kaust.edu.sa
${ }^{1}$ T. Jungwirth, J. Sinova, J. Mašek, J. Kučera, and A. H. MacDonald,
Rev. Mod. Phys. 78, 809 (2006).
${ }^{2}$ K. Sato, L. Bergqvist, J. Kudrnovský, P. H. Dederichs, O. Eriksson,
I. Turek, B. Sanyal, G. Bouzerar, H. Katayama-Yoshida, V. A. Dinh,
T. Fukushima, H. Kizaki, and R. Zeller, Rev. Mod. Phys. 82, 1633
(2010).

${ }^{3}$ K. S. Novoselov, A. K. Geim, S. V. Morozov, D. Jiang, Y. Zhang, S. V. Dubonos, I. V. Grigorieva, and A. A. Firsov, Science 306, 666 (2004).

${ }^{4}$ D. J. Priour, E. H. Hwang, and S. Das Sarma, Phys. Rev. Lett. 95, 037201 (2005).

${ }^{5}$ E. Z. Meilikhov and R. M. Farzetdinova, Phys. Rev. B 74, 125204 (2006).

${ }^{6}$ A. V. Krasheninnikov, P. O. Lehtinen, A. S. Foster, P. Pyykkö, and R. M. Nieminen, Phys. Rev. Lett. 102, 126807 (2009).

${ }^{7}$ B. Huang, H. Xiang, J. Yu, and S.-H. Wei, Phys. Rev. Lett. 108, 206802 (2012).

${ }^{8}$ Y. Kim, J.-L. Huang, and C. M. Lieber, Appl. Phys. Lett. 59, 3404 (1991).

${ }^{9}$ J. Heising and M. G. Kanatzidis, J. Am. Chem. Soc. 121, 11720 (1999).

${ }^{10}$ K. F. Mak, C. Lee, J. Hone, J. Shan, and T. F. Heinz, Phys. Rev. Lett. 105, 136805 (2010).

${ }^{11}$ A. Kuc, N. Zibouche, and T. Heine, Phys. Rev. B 83, 245213 (2011).

${ }^{12}$ D. Xiao, G.-B. Liu, W. Feng, X. Xu, and W. Yao, Phys. Rev. Lett. 108, 196802 (2012).

${ }^{13}$ B. Radisavljevic, J. Brivio, V. Giacometti, and A. Kis, Nat. Nanotechnol. 6, 147 (2011)

${ }^{14}$ J. N. Coleman, M. Lotya, A. O’Neill, S. D. Bergin, P. J. King, U. Khan, K. Young, A. Gaucher, S. De, R. J. Smith, I. V. Shvets, S. K. Arora, G. Stanton, H.-Y. Kim, K. Lee, G. T. Kim, G. S. Duesberg, T. Hallam, J. J. Boland, J. J. Wang, J. F. Donegan, J. C. Grunlan, G. Moriarty, A. Shmeliov, R. J. Nicholls, J. M. Perkins, E. M. Grieveson, K. Theuwissen, D. W. McComb, P. D. Nellist, and V. Nicolosi, Science 331, 568 (2011).
${ }^{15}$ Y.-H. Lee, X.-Q. Zhang, W. Zhang, M.-T. Chang, C.-T. Lin, K.-D. Chang, Y.-C. Yu, J. T.-W. Wang, C.-S. Chang, L.-J. Li, and T.-W. Lin, Adv. Mater. 24, 2320 (2012).

${ }^{16}$ P. Giannozzi, S. Baroni, N. Bonini, M. Calandra, R. Car, C. Cavazzoni, D. Ceresoli, G. L. Chiarotti, M. Cococcioni, I. Dabo, A. D. Corso, S. de Gironcoli, S. Fabris, G. Fratesi, R. Gebauer, U. Gerstmann, C. Gougoussis, A. Kokalj, M. Lazzeri, L. Martin-Samos, N. Marzari, F. Mauri, R. Mazzarello, S. Paolini, A. Pasquarello, L. Paulatto, C. Sbraccia, S. Scandolo, G. Sclauzero, A. P. Seitsonen, A. Smogunov, P. Umari, and R. M. Wentzcovitch, J. Phys.: Condens. Matter 21, 395502 (2009).

${ }^{17}$ R. M. Martin, Electronic Structure: Basic Theory and Practical Methods (Cambridge University Press, Cambridge, UK, 2004).

${ }^{18}$ D. Vanderbilt, Phys. Rev. B 41, 7892 (1990).

${ }^{19}$ J. P. Perdew, K. Burke, and M. Ernzerhof, Phys. Rev. Lett. 77, 3865 (1996).

${ }^{20}$ Y. C. Cheng, Z. Y. Zhu, and U. Schwingenschlögl, RSC Adv. 2, 7798 (2012).

${ }^{21}$ Z. Y. Zhu, Y. C. Cheng, and U. Schwingenschlögl, Phys. Rev. B 84, 153402 (2011)

${ }^{22}$ T. Cheiwchanchamnangij and W. R. L. Lambrecht, Phys. Rev. B 85, 205302 (2012).

${ }^{23}$ F. L. Deepak, R. Esparza, B. Borges, X. Lopez-Lozano, and M. Jose-Yacaman, ACS Catal. 1, 537 (2011).

${ }^{24}$ H. T. Wang, Q. X. Wang, Y. C. Cheng, K. Li, Y. B. Yao, Q. Zhang, C. Z. Dong, P. Wang, U. Schwingenschlögl, W. Yang, and X. X. Zhang, Nano Lett. 12, 141 (2012).

${ }^{25}$ H.-P. Komsa, J. Kotakoski, S. Kurasch, O. Lehtinen, U. Kaiser, and A. V. Krasheninnikov, Phys. Rev. Lett. 109, 035503 (2012).

${ }^{26}$ J. J. Palacios and F. Ynduráin, Phys. Rev. B 85, 245443 (2012).

${ }^{27}$ J. Kudrnovský, I. Turek, V. Drchal, F. Máca, P. Weinberger, and P. Bruno, Phys. Rev. B 69, 115208 (2004).

${ }^{28}$ F. Máca, J. Kudrnovský, V. Drchal, and G. Bouzerar, Appl. Phys. Lett. 92, 212503 (2008) 